\title{
Homocystiene and C-Reactive Protein in Detection of Frailty
}

\author{
Moatassem S. Amer1, Tamer M. Farid1, Ekrami E. Abd El-Rahman', \\ Deena M. EL-Maleh1* ${ }^{*}$, Omar H. Omar², Randa A. Mabrouk ${ }^{3}$ \\ ${ }^{1}$ Geriatrics and Gerontology Department, Faculty of Medicine, Ain Shams University, Cairo, Egypt \\ ${ }^{2}$ Radiodiagnosis Department, Faculty of Medicine, Ain Shams University, Cairo, Egypt \\ ${ }^{3}$ Clinical Pathology Department, Faculty of Medicine, Ain Shams University, Cairo, Egypt \\ Email: doc dodi80@yahoo.com
}

Received 10 February 2014; revised 8 April 2014; accepted 24 April 2014

Copyright (C) 2014 by authors and Scientific Research Publishing Inc.

This work is licensed under the Creative Commons Attribution International License (CC BY).

http://creativecommons.org/licenses/by/4.0/

(c) (7) Open Access

\begin{abstract}
Background and Aim of the Work: Frailty is a state of reduced physiological reserve and is associated with increased susceptibility to disability. It is associated with a high morbidity and mortality. The aim of this work is to assess the association between the levels of homocystiene and C-reactive protein and frailty and to examine the ability of homocystiene as a new marker to detect frailty. Subjects and Methods: A total number of 104 elderly subjects (above 60 years old) were included in this study and they were subjected to history, examination, comprehensive geriatric assessment, and laboratory investigations including: C-reactive protein (CRP) and Homocystiene (Hcy). The patients were divided into frail and non-frail groups using Fried's criteria as applied by AvilaFunes et al., 2008 and each group included 52 patients. Results: There was no significant difference between the two groups as regards age gender or smoking habits. But there were higher levels of CRP ( $>10 \mathrm{mg} / \mathrm{dl})$ and homocystiene ( $>12 \mathrm{mmol} / \mathrm{l})$ among frail cases and by comparing both homocystiene was more sensitive in detection of frailty. Conclusion: Frailty was associated with eleveted homocystiene and CRP and homocystiene is more specific than CRP in detection of frailty.
\end{abstract}

\section{Keywords}

Frailty, CRP, Homocystiene

\section{Introduction}

Frailty is becoming increasingly common as the world's population ages. There is no single best definition of frailty but the most acceptable definition is postulated by Fried et al. defined frailty as: "A physiologic syn-

${ }^{*}$ Corresponding author. 
drome characterized by decreased reserve and resistance to stressors, resulting from cumulative decline across multiple physiologic systems, and causing vulnerability to adverse outcomes” [1]. Different conceptual approaches have been applied to describe this phenomenon, including incorporation of physical characteristics and function [2], and utilizing a combination of clinical deficits and comorbidity domain [3].

Lang et al. [4] reported that clinical markers or indicators are insufficient to differentiate the frailty process from normal aging, and they gave rise to the necessity to detect frailty at a pre-clinical stage with the help of biomarkers. Evaluation of alterations in human biomarkers and their relationships to differing models of frailty may assist the determination of the initiation of the processes that eventually led to frailty [5].

Homocysteine is one possible candidate that may underlie the development of the frailty syndrome. Total plasma homocystiene has been shown to be inversely related to the intake and plasma levels of folate and B-vitamins. The deficiencies of B-vitamins may result in mitochondrial dysfunction with deleterious changes in cellular function. These could conceivably cause muscle weakness and atrophy, leading to sarcopenia with progressive physical decline. At the molecular level, B-vitamin deficiency may be mediated via hyperhomocysteinemia through mechanisms of oxidative stress, or by homocysteinylation, which involves covalent binding of homocystiene to proteins. These modified proteins can trigger the inflammatory cascade, resulting in vascular endothelium damage and subsequently vascular events, further leading to functional decline and frailty [6].

Older frail patients exhibit evidence of increased inflammation, with higher levels of C-reactive protein [7]. A direct role of inflammation in the development of frailty is primarily based on the catabolic effects that proinflammatory cytokines have on muscles. TNF-a and other inflammatory signals increase IL-6 production which in turn stimulates production of CRP: all are markers of the same inflammatory process and each has detrimental effects on muscle [8]. Elevated CRP levels are also associated with many late-life chronic conditions, including Alzheimer's disease, cardiovascular diseases, macular degeneration, and functional decline, disability, as well as all-cause mortality in older adults [9]-[11].

In this study, the researchers sought to determine if elevated homocystiene is associated with frailty in elderly, (as the role of homocystiene in frailty was not thoroughly investigated) and to assess whether it is better in detection of frailty than CRP (whose role in frailty has been investigated by many researchers but it is linked to many other chronic conditions) so that when homocystiene is used it can increase the sensitivity of the physical criteria in detection of frailty.

\section{Patients and Methods}

\subsection{Study Design and Setting}

The study is a Case-control study it included 104 Elderly participants (60 years old and above), both males and females were recruited from Ain Shams University hospital from inpatient wards and outpatient clinics from January 2011 till December 2012. One hundred and twenty participants were interviewed 16 were excluded 10 of them had renal impairment by labs (which lead to increased Homocystiene level) 8 were excluded due to technical difficulties. They were divided into two groups which are:

Cases Group: 52 frail elderly 60 years and older diagnosed by Fried's criteria [12] as applied by Avila-Funes et al. [13]. The participants were considered to be "frail" if they had three or more frailty components among the five criteria.

Controls Group: 52 elderly 60 years and older matched with cases regarding age and gender. They are not frail or have 2 or less of frailty criteria.

\subsection{Data Collection}

Any patient who refused to participate in the study, patients who were suffering from acute infection, and any patients who were taking drugs that have antiinflammatory effects as steroids, statins and aspirin were excluded from this study.

Each patient then underwent comprehensive geriatric assessment in the form of detailed history and physical examination, cognitive function assessment by Minimental status examination (MMSE) [14] (The Arabic version used in this study was done by El-Okl et al. [15], functional assessment by Activities of daily living (ADL) [16], Arabic version [17], and Instrumental activities of daily living (IADL) [18] (An Arabic version of the test 
was applied [19], and Geriatric depression scale 15 items (GDS-15) [20] was used to screen for depression the Arabic version of the test was applied by Shehta et al. [21] (Those items are done as a routine assessment of elder patients).

Frailty was defined according to the construct previously validated by Fried et al. in the Cardiovascular Health Study [12]. All five components from the original phenotype were retained; however, the metrics used to characterize the frailty criteria were slightly different and defined as follows [13]:

- Shrinking - Recent and unintentional weight loss of $\geq 3 \mathrm{~kg}$ in the prior year was identified and body mass index calculated. Participants who answered "yes" for weight loss or had a body mass index $<21 \mathrm{~kg} / \mathrm{m}^{2}$ were considered to be frail for this component.

- Poor endurance and energy - As indicated by self report of exhaustion, identified by two questions from the Center for Epidemiological Studies-Depression scale (CES-D) [22]: "I felt that everything I did was an effort" and "I could not get going." Participants were asked: "How often, in the last week, did you feel this way?" 0 $=$ rarely or none of the time; $1=$ some or a little of the time; $2=$ a moderate amount of the time; or $3=$ most of the time. Participants answering " 2 " or " 3 " to either of these questions were considered as frail by exhaustion.

- Slowness-Meets criteria for frailty if time to walk $6 \mathrm{~m}$ was $\geq 8$ seconds for height $\leq 173 \mathrm{~cm}$ or $>7$ seconds for height $>173 \mathrm{~cm}$ in males, and $\geq 8$ seconds for height $\leq 159 \mathrm{~cm}$ or $>7$ seconds for height $>159 \mathrm{~cm}$ in females.

- Weakness-Participants answering "yes" to the following question were categorized as frail for this component: "Do you have difficulty rising from a chair?"

- Low physical activity-A single response was used to estimate physical activity. Individuals who denied doing daily leisure activities such as walking or gardening and/or denied doing some sport activity per week were categorized as physically inactive. Those who reported doing them were considered to be active.

- Patients who had 3 of the 5 items were diagnosed as frail.

\subsection{Laboratory Investigations}

Six mL whole blood was drawn from each participant after 12 hours fasting, and was divided into 2 tubes: $3 \mathrm{~mL}$ in EDTA-anticoagulated tube for homocystiene assay Plasma was separated from the blood samples within 1 hour of collection and stored at $-80^{\circ} \mathrm{C}$ until assayed. The other $3 \mathrm{~mL}$ were allowed to clot in a plain tube and the resulting serum was used for quantitative c-reactive protein. Homocystiene was assayed by Enzyme immunoassay (EIA) kit: Axis ${ }^{\circledR}$ Homocysteine EIA (Axis-Shield Diagnostics Ltd, The Technology Park, Dundee DD2 1XA,United Kingdom) Hyperhomocysteinaemia was defined as levels greater than $12 \mathrm{mmol} / \mathrm{l}$ [23]. Quantitation of CRP was performed by immunoturbidimetric assay using Biosystems CRP-hs kit (Biosystems, SA, Barcelona, Spain) CRP level in normal healthy adults is usually low $<10 \mathrm{mg} / \mathrm{dl}$ [24].

\section{Data Management}

Analysis of data was performed by using the 12th version of Statistical Package for Social Science (SPSS). Description of all data in the form of mean (M) and standard deviation (SD) for all quantitative variables was done. Frequency and percentage was done for all qualitative variables. Comparison between quantitative variables was done using t-test to compare to compare two groups and ANOVA (analysis of variance) to compare more than two groups. Comparison of qualitative variables was done using the Chi-square test. Correlation coefficient also was used to find linear relation between different variables using r-test or Sperman correlation co-efficient. Significant level measured according to $\mathrm{P}$ value (probability), $\mathrm{P}>0.05$ is insignificant, $\mathrm{P}<0.05$ is significant and $\mathrm{p}$ $<0.01$ is highly significant.

\section{Results}

As regards demographic criteria of the study population, there was no significant difference between cases and controls as regards: age, gender, living arrangement and smoking habits.

Regarding clinical characteristics frail patients had higher levels of ADL and IADL dependence in addition to higher grades of depression and cognitive impairment. There was a higher mean number of associated chronic disease among frail elderly. Table 1 shows the distribution of chronic disease among frail and non frail partici- 
Table 1. Comparison between the two studied groups as regards chronic diseases.

\begin{tabular}{|c|c|c|c|c|c|c|}
\hline & \multicolumn{2}{|c|}{ Cases } & \multicolumn{2}{|c|}{ Controls } & \multirow{2}{*}{$\mathrm{T}$} & \multirow{2}{*}{ P-value } \\
\hline & No. & $\%$ & No. & $\%$ & & \\
\hline Diabetes mellitus & 24 & 46.2 & 14 & 26.9 & 4.1 & $0.04^{*}$ \\
\hline IHD & 28 & 53.8 & 9 & 17.3 & 15.1 & $0.000^{* *}$ \\
\hline Hypertension & 26 & 50.0 & 14 & 26.9 & 5.8 & $0.01^{*}$ \\
\hline Stroke & 10 & 19.2 & 2 & 3.8 & 6.0 & $0.01^{*}$ \\
\hline COPD & 18 & 34.6 & 19 & 36.5 & 0.4 & 0.8 \\
\hline Arthritis & 20 & 38.5 & 18 & 34.6 & 0.1 & 0.6 \\
\hline Visual impairment & 40 & 76.9 & 18 & 34.6 & 18.8 & $0.000^{* *}$ \\
\hline Hearing impairment & 8 & 15.4 & 0 & 0 & 8.6 & $0.003^{* *}$ \\
\hline Chronic liver disease & 10 & 19.2 & 5 & 9.6 & 1.9 & 0.1 \\
\hline Thyroid disease & 6 & 11.5 & 0 & 0 & 6.3 & $0.01^{*}$ \\
\hline Anemia & 4 & 7.7 & 0 & 0 & 4.1 & 0.06 \\
\hline
\end{tabular}

${ }^{*} \mathrm{P}<0.05$ significant, ${ }^{* *} \mathrm{P}<0.01$ highly significant.

pants there was higher percentage of diabetes mellitus (DM), Ischemic heart disease(IHD), hypertension, stroke, visual and hearing impairment among cases. The three most prevalent chronic illnesses among cases were visual impairment, DM, and IHD (the least common were hearing impairment, stroke and chronic liver disease). As for controls the three most prevalent chronic illnesses were chronic obstructive pulmonary disease (COPD), Visual impairment and arthritis.

Our study showed that there was higher mean CRP \& homocystiene levels among frail cases compared to controls and the difference is significant statistically as shown in Table 2.

Finally by comparing the sensitivity and specificity of homocystiene and CRP in detection of frailty homocystiene was found to be more sensitive than CRP as it had a higher area under the curve than CRP as shown in Figure 1 and Figure 2.

\section{Discussion}

Frailty has been recognized as a common clinical syndrome associated with a high rate of morbidity and mortality [25].

The current study assessed the association between the levels of homocystiene and C-reactive protein and frailty and to examine the ability of homocystiene as a new marker to detect frailty. Applying routine comprehensive geriatric assessments showed that frail patients had had higher levels of ADL and IADL dependence in addition to higher grades of depression and cognitive impairment and also a higher mean number of associated chronic diseases which was consistent with Espinoza et al. 2007 who stated that frailty is associated with multiple impairment and comorbidities [26].

The association between raised markers of inflammation and frailty has been searched in several studies as in our study we found that frail elderly had higher levels of CRP in comparison to non-frail controls Walston et al. 2002 found in results from Cardiovascular Health Study (CHS) that higher CRP levels are associated with higher frailty risk [7] \& this was also approved by Hubbard et al. 2009 [8]. These data support the hypothesis of the role of inflammation in the development of frailty.

Homocystiene level was higher in cases than controls \& the differences were statistically significant which agrees with results of Wong et al. 2013 that hyperhomocysteinemia is associated with the prevalence of frailty. It is also predictive of all-cause mortality independent of frailty. The study by Wong et al. included 4248 participants. One thousand one hundred-seventeen participants had high plasma total homocysteine levels of 15 micromoles per liter or more. Six hundred eighty-five subjects were categorized as frail. Among subjects who had high homocysteine levels, the adjusted risk of frailty as assessed during was $49 \%$ greater than those whose homocysteine was less than 15 micromoles per liter [6]. It also agrees to the results of Bates et al. 2010 that high homocystiene is also a robust and independent predictor of subsequent mortality in these older adults, both for 
Table 2. Comparision between cases \& controls as regards Homocystiene \& CRP.

\begin{tabular}{|c|c|c|c|c|c|}
\hline & \multicolumn{2}{|c|}{ Cases } & \multicolumn{2}{|c|}{ Controls } & \multirow{2}{*}{$\mathrm{P}$} \\
\hline & Mean & SD & Mean & SD & \\
\hline CRP & 31.3 & 38.9 & 14.4 & 13.7 & $0.004^{* *}$ \\
\hline Homocystiene & 15.4 & 10.3 & 6.8 & 6.8 & $0.000^{* *}$ \\
\hline
\end{tabular}

${ }^{*} \mathrm{P}<0.05$ significant, ${ }^{* *} \mathrm{P}<0.01$ highly significant.

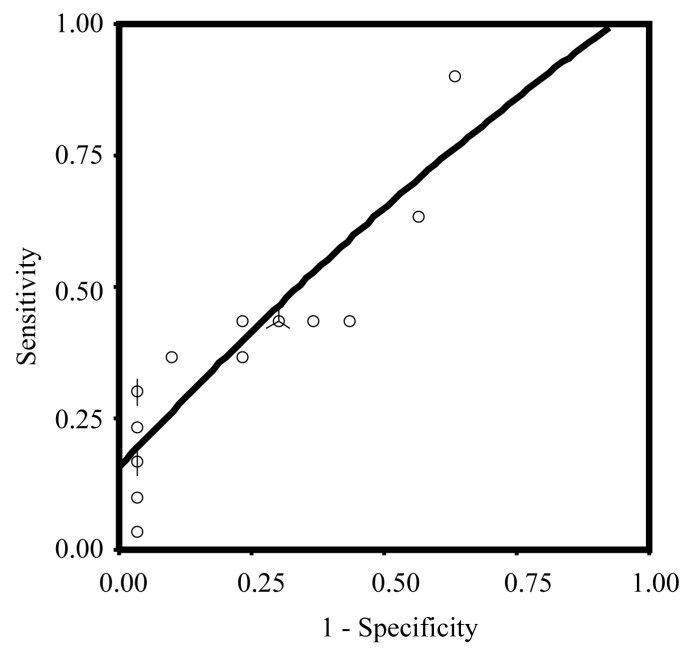

Figure 1. CRP area under the curve (0.65).

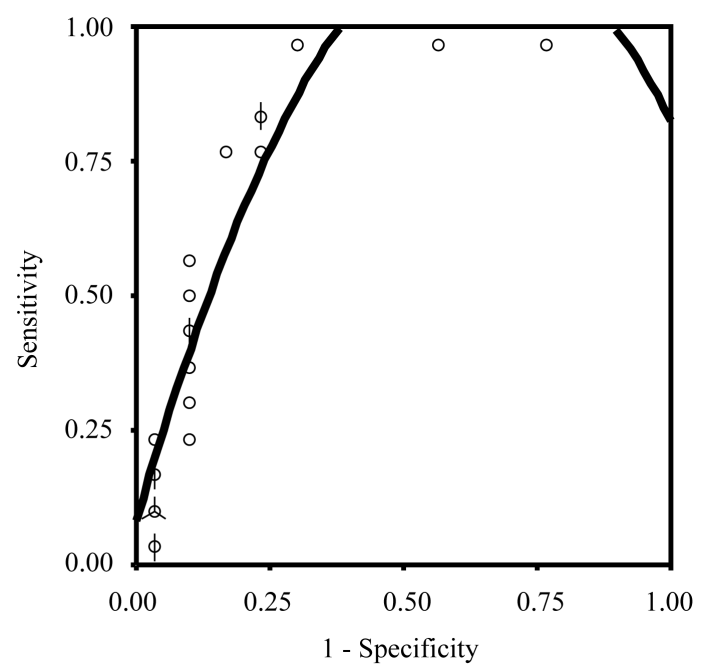

Figure 2. Homocystiene area under the curve (0.85).

all-cause mortality and especially for primary-cause vascular mortality [27]. These results support the hypothesized role of homocysteine in the development of frailty.

By comparing the sensitivity \& specificity of CRP \& homocystiene in the detection of frailty the latter was found to be better predictor of frailty, contrary to Wong et al. 2013 who stated that homocysteine \& CRP are both associated with frailty but independent from each other. (The hypothesis of Hcy induced inflammation as a mechanism of physical decline was tested when high-sensitivity C-reactive protein was added to the fully adjusted model for frailty [6]. The effect estimates were altered minimally, implying that the relationship between Hcy and frailty in this cohort may be independent of the inflammatory pathway). Houwelingen et al. 2013 stated that CRP \& homocystiene are equally related to mortality in elderly (85 years \& older) [28]. 


\section{Conclusion}

Frailty is an important condition among the elderly but it needs further studies regarding risk factors and associations. The significant elevation of CRP (which is an inflammatory marker) in frail elderly, may give support to the theory of inflammaging. Also the increase in homocystiene level and its higher specificity for frailty than CRP suggest it might be used as a marker for frailty which can be used with Fried's clinical criteria to detect frailty. Lowering homocystiene level using vitamin B12 may affect the progression of this state. However this matter needs to be investigated in the future.

\section{References}

[1] Fried, L.P. and Walston, J. (2003) Frailty and Failure to Thrive. In: Hazzard, W., Blass, J.P., Halter, J.B., et al., Eds., Principles of Geriatric Medicine and Gerontology, 5th Edition, McGraw-Hill, New York, 1487-1502.

[2] Fried, L.P., Tangen, C.M., Walston, J., et al. (2001) Cardiovascular Health Study Collaborative Research Group. Frailty in Older Adults: Evidence for a Phenotype. The Journals of Gerontology Series A: Biological Sciences and Medical Sciences, 56, M146-M156. http://dx.doi.org/10.1093/gerona/56.3.M146

[3] Jones, D., Song, X., Mitnitski, A., et al. (2005) Evaluation of A Frailty Index Based on a Comprehensive Geriatric Assessment in a Population Based Study of Elderly Canadians. Aging Clinical and Experimental Research, 17, 465-471. http://dx.doi.org/10.1007/BF03327413

[4] Lang, P., Drame, M., Jolly, D., et al. (2011) Frailty: Learnings from the Cohort Study and Future Perspectives for the Research. Geriatric Psychiatry New Psychiatrvicility, 9, 135-149.

[5] Williams, J.E.R. (2010) Characteristics of Frailty in Community. Dwelling Eldes, PhD Dissertation, The Ohio State University, 83.

[6] Wong, Y., Almeida, O., Mc Caul, K., et al. (2013) Homocysteine, Frailty, and All-Cause Mortality in Older Men: The Health in Men Study. The Journals of Gerontology Series A: Biological Sciences and Medical Sciences, 68, 590-598. http://dx.doi.org/10.1093/gerona/gls211

[7] Walston, J., Mc Burnie, M.A., Newman, A., et al. (2002) Cardiovascular Health Study. Frailty and Activation of the Inflammation and Coagulation Systems with and without Clinical Comorbidities: Results from the Cardiovascular Health Study. Archives of Internal Medicine, 162, 2333-2341. http://dx.doi.org/10.1001/archinte.162.20.2333

[8] Hubbard, R.E., Searle, S.D., Mitnitski, A. and Rockwood, K. (2009) Effect of Smoking on the Accumulation of Deficits, Frailty and Survival in Older Adults: A Secondary Analysis from the Canadian Study of Health and Aging. The Journal of Nutrition, Health \& Aging, 13, 468-472. http://dx.doi.org/10.1007/s12603-009-0085-y

[9] Harris, T.B., Ferrucci, L., Tracy, R.P., et al. (1999) Associations of Elevated Interleukin-6 and C-Reactive Protein Levels with Mortality in the Elderly. American Journal of Medicine, 106, 506-512. http://dx.doi.org/10.1016/S0002-9343(99)00066-2

[10] Taaffe, D.R., Harris, T.B., Ferrucci, L., et al. (2000) Cross-Sectional and Prospective Relationships of Interleukin-6 and C-Reactive Protein with Physical Performance in Elderly Persons: Mac Arthur Studies of Successful Aging. The Journals of Gerontology Series A: Biological Sciences and Medical Sciences, 55, M709-M715. http://dx.doi.org/10.1093/gerona/55.12.M709

[11] Hage, F.G. and Szalai, A.J. (2007) C-Reactive Protein Gene Polymorphisms, C-Reactive Protein Blood Levels, and Cardiovascular Disease Risk. Journal of the American College of Cardiology, 18, 1115-1122.

[12] Fried, L., Kronmal, R., Newman, A., et al. (1998) Risk Factors for 5 Year Mortality in Older Adults: The Cardiovascular Health Study. The Journal of the American Medical Association, 279, 585-592. http://dx.doi.org/10.1001/jama.279.8.585

[13] Avila-Funes, J.A., Helmer, C., Amieva, H., et al. (2008) Frailty among Community-Dwelling Elderly People in France: The Three-City Study. Journal of Gerontology: Medical Sciences by the Gerontological Society of America, 63A, 1089-1096.

[14] Folstein, M., Folstein, S. and Mc Hug, P. (1975) Minimentalstate. A Practical Method for Gradient the Cognitive State of Patients for Clinicians. Journal of Psychiatric Research, 12, 189-198. http://dx.doi.org/10.1016/0022-3956(75)90026-6

[15] El-Okl, M. (2002) Prevalence of Alzheimer Dementia and Other Causes of Dementia in Egyptian Elderly. Dissertation, Ain Shams University, Cairo.

[16] Katz, S., Ford, A., Moswowitz, R., Jackson, B. and Jaffe, M. (1963) Studies of Illness in the Aged. The Index of ADL: A Standardized Measure of Biological and Psychological Function. The Journal of the American Medical Association, 185, 914-919. http://dx.doi.org/10.1001/jama.1963.03060120024016 
[17] El-Sherpiny, M., Mortagy, A. and Fahy, H. (2000) Prevalence of Hypercholesterolemia among Elderly People Living in Nursing Houses in Cairo. Geriatric Department Library, Ain Shams University, Cairo, 6.

[18] Lawton, M. and Brody, E. (1969) Assessment of Older People: Self-Maintaining and Instrumental Activities of Daily Living. The Gerontologist, 9, 179-186. http://dx.doi.org/10.1093/geront/9.3_Part_1.179

[19] Fillenbaum, G. (1986) The Wellbeing of the Elderly: Approaches to Multi-Dimensional Assessment. World Health Organization, WHO Offset Publication No. 84, Arabic Translation Distributed by the Eastern Mediterranean Regional Office.

[20] Sheikh, J.A. and Yesavage, J.I. (1986) Geriatric Depression Scale (GDS): Recent Evidence and Development of a Shorter Version. Clinical Gerontologist, 5, 165-173. http://dx.doi.org/10.1300/J018v05n01_09

[21] Shehta, A.S., El-Banouby, M.H., Mortagy, A.K., et al. (1998) Prevalence of Depression among Egyptian Geriatric Community. Geriatric Department Library, Ain ShamsUniversity, Cairo, 3-5.

[22] Orme, J., Reis, J. and Herz, E. (1986) Factorial and Discriminate Validity of the Center for Epidemiological Studies Depression (CES-D) Scale. Journal of Clinical Psychology, 42, 28-33. http://dx.doi.org/10.1002/1097-4679(198601)42:1<28::AID-JCLP2270420104>3.0.CO;2-T

[23] Friedman, A.N., Bostom, A.G., Selhub, J., Levey, A.S. and Rosenberg, I.H. (2001) The Kidney and Homocysteine Metabolism. Journal of the American Society of Nephrology, 12, 2181-2189.

[24] Amos, B., Bateman, N., Dakin, D., et al. (2010) C-Reactive Protein (CRP) Information and Resources. WebMD Medical Reference from Healthwise.

[25] Inaki, M., Iturbe, A., Gome, J., et al. (2010) The Frail Elderly, Detection and Treatment. Aten Primaria, 42, 388-390.

[26] Espinoza, S. and Fried, L. (2007) Risk Factors for Frailty in Older Adults. Clinical Geriatrics, 15, 37-44.

[27] Bates, C.J., Mansoor, M.A., Pentieva, K.D., Hamera, M. and Mishra, G.D. (2010) Biochemical Risk Indices, Including Plasma Homocysteine, that Prospectively Predict Mortality in Older British People: The National Diet and Nutrition Survey of People Aged 65 Years and Over. British Journal of Nutrition, 104, 893-899. http://dx.doi.org/10.1017/S0007114510001236

[28] Van Houwelingen, A.H., den Elzen, W.P., Mooijaart, S.P., Heijmans, M., Blom, J.W., de Craen, A.J. and Gussekloo, J. (2013) Predictive Value of a Profile of Routine Blood Measurements on Mortality in Older Persons in the General Population: The Leiden 85-Plus Study. PLoS ONE, 8, Article ID: e58050. 